\title{
Ni solas ni resignadas: la participación femenina en las actividades científico-académicas de la Argentina en los inicios del siglo XX"
}

\begin{abstract}
Susana V. García**
Resumen

Alrededor de 1910 se registran los primeros trabajos de investigación en ciencias naturales firmados por mujeres en las publicaciones científicas argentinas, egresan de las universidades las primeras doctoras en estas disciplinas y las mujeres son incorporadas como ayudantes en laboratorios, museos $y$ reparticiones públicas. En este trabajo se analiza el contexto de emergencia de esa primera generación de mujeres que participaron formalmente en el ámbito científico-institucional argentino. En primer lugar se examina el acceso femenino a la educación universitaria y los estudios científicos, y luego, las inserciones institucionales, becas y trabajos de algunas de las primeras doctoras y profesoras en ciencias naturales.
\end{abstract}

Palavras-chave: Mujeres, Universidad, Ciencias Naturales, Museos.

\footnotetext{
* Recebido para publicação em maio de 2006, aceito em agosto de 2006. [Revisão Martha Ramirez.] Parte de los materiales aquí utilizados proceden del Archivo Histórico, la Biblioteca de la Facultad de Ciencias Naturales y el Museo de La Plata. Especialmente agradezco la colaboración prestada en la búsqueda bibliográfica al personal de la Biblioteca de la Facultad de Humanidades y Ciencias de Educación de La Plata y a la Lic. Andrea Pegoraro, también a la Dra. María Jesús Santemases por haberme facilitado sus trabajos, a la Dra. Cecilia von Reichenbach, así como a la Dra. Irina Podgorny por la lectura del borrador de este trabajo.

** Becaria Postdoctoral CONICET, Archivo Histórico del Museo de La Plata. garcia_su@yahoo.com.ar
}

cadernos pagu (27), julho-dezembro de 2006: pp.133-172. 
Ni solas ni resignadas

Neither Lonely nor Resigned: Women's participation in Scientific and Academic Activities in Argentina in the Begining of the $20^{\text {th }}$ century

\begin{abstract}
Around 1910, the first papers in natural sciences signed by women were published in the Argentinean scientific review, the women took the doctoral's degrees in these disciplines and several were hired as assistants in laboratories, museums and public distributions. In this article, we analyse the context of emergency of this first generation of women who participated formally in the Argentinean scientific-academic setting. Firstly, we examine the feminine access to the university and the scientific studies; and then, the institutional jobs, scholarships and works of some of the first graduated women in natural sciences.
\end{abstract}

Key Words: Women, University, Natural Sciences, Museums. 
En la historiografía de las ciencias argentinas, la cuestión de género no ha constituido un campo de indagación particular y son poco frecuentes los trabajos que se detienen en la situación de la mujer en la actividad científica local. En algunos casos se ha tendido a rescatar del "olvido" a ciertas figuras consideradas mujeres "heroicas" o "pioneras" en el campo de la medicina, la producción intelectual o científica. No obstante, este tipo de relato biográfico corre el riesgo de retornar a una vieja tradición historiográfica basada en "la historia de los héroes", o en este caso "las grandes mujeres", que contribuye poco en la comprensión de los procesos históricos. (Bianchi, 1992:17-33) Por otro lado, puede conducir a crear un cuadro distorsionado o una imagen estereotipada, donde las trayectorias individuales muestran a unas pocas mujeres "excepcionales" enfrentadas a un ambiente hostil y transponiendo las "reglas" de la "exclusión femenina". En ese sentido, por lo general las historias centradas en las "precursoras" enfatizan en las barreras y obstáculos que encontraron esas mujeres, pero, en cambio, ofrecen poca información acerca de las prácticas científicas concretas en las que participaron, los cambios operados en la organización social e institucional del trabajo científico y los factores - familiares, sociales, económicos, educacionales, personales - que permitieron la presencia femenina. En la medida en que las historias de vida individuales se articulen con los procesos sociales y "en relación" con otros actores, contextos instituciones y prácticas científicas, los relatos biográficos puede aportar información sobre los constreñimientos y la organización de la actividad científica en determinados contextos o la participación de ciertos sectores o actores no contemplados en la historiografía oficial. De esta forma, analizar itinerarios científicos femeninos o la participación de las mujeres en la empresa científica, permite remitirnos a algo más que al resultado de la voluntad de agentes individuales: el carácter colectivo y social de la ciencia y la materialidad de las prácticas científicas concretas. Desde esta perspectiva, identificar el papel o los papeles desempeñados por las mujeres en la organización de 
Ni solas ni resignadas

las actividades científicas cotidianas, sus trabajos y estrategias, constituye un intento de construir un panorama más complejo del desarrollo de la empresa científica.

En este trabajo nos centraremos en la participación femenina en el campo de las ciencias naturales en las primeras décadas del siglo XX. En el contexto argentino de 1910 se registran los primeros trabajos de investigación firmados por mujeres en las publicaciones científicas locales, egresan de las universidades las primeras doctoras en estas disciplinas y las mujeres son incorporadas como ayudantes y auxiliares en laboratorios, museos, observatorios y oficinas de ciencia estatal. El análisis de la composición del estudiantado, los becados y las trayectorias de los primeros graduados en ciencias naturales permite observar que la participación femenina alcanzó un porcentaje más significativo de lo que generalmente se presupone $\mathrm{y}$, al mismo tiempo, también habla de un campo débil en recursos humanos para afrontar la expansión institucional y especialización que se opera durante las primeras décadas del siglo XX. Por otro lado, los trabajos científicos y las inserciones instituciones de algunas de ellas remiten a un entramado de contactos personales y vínculos familiares, estereotipos de trabajo femenino, disputas de poder, criterios de reconocimiento y selección de personal, entre otros aspectos vinculados al ámbito científico-académico. Al mismo tiempo, es factible observar cómo se integraron a las redes de relaciones académicas y extra-académicas que permitía que especimenes de diferentes regiones llegaran a sus mesas de trabajo, contar con apoyo logístico para recolección y observación en el campo, así como participar en los circuitos de intercambio de información y material de estudio. En las siguientes páginas se procura, entonces, integrar el análisis de las trayectorias de las primeras egresadas en ciencias naturales y otras mujeres que publicaron trabajos de investigación en estas disciplinas en el marco del acceso femenino a la educación superior y los espacios institucionales de las ciencias naturales en la Argentina de principios del siglo XX. 
El acceso de las mujeres a la educación universitaria y los estudios científicos

La última parte del siglo XIX registra la presencia de algunas alumnas y las primeras diplomadas en las universidades argentinas, junto con mayores oportunidades de educación postescolar para las mujeres y sectores de menores recursos. Durante la década de 1870 se organizaron y expandieron las escuelas normales para la formación de maestros y maestras en distintas ciudades del país. Para la organización de estas instituciones se contrataron maestras norteamericanas, algunas de las cuales promovieron entre sus alumnas el interés por seguir estudios universitarios, la coeducación y la reivindicación del papel y los derechos de mujer en la sociedad. Las escuelas normales constituyeron una experiencia exitosa de educación secundaria pública para las mujeres y sectores con menores recursos. En la transición del siglo XIX al XX, si bien en algunos colegios nacionales, donde se otorgaba el título de bachiller requerido para el ingreso a la universidad, aceptaron la inscripción de mujeres, especialmente a las hijas de los profesores o de la elite política o intelectual local, el acceso de las mujeres a estas instituciones no era algo generalizado y en algunos casos pudieron obtener el diploma de bachiller rindiendo libre los exámenes. En general, el alto costo de los estudios en estos establecimientos y su perfil elitista y masculino, constituyó un importante mecanismo de segmentación social a nivel de la educación media. Hasta las primeras décadas del siglo $\mathrm{XX}$, cuando se produce una mayor diversificación de las instituciones educativas, los grupos con memores recursos y las mujeres tendieron a concentrarse en el circuito educativo conformado por las escuelas de enseñanza primaria o elemental y las escuelas normales, donde se formaban los maestros y profesores para estos mismos establecimientos, mientras que el otro circuito de formación y reclutamiento de docentes se integraba a través del colegio nacional (bachillerato)- 
Ni solas ni resignadas

universidad. ${ }^{1}$ En general, al iniciarse el siglo XX la matriculación de los/las egresados/as de las escuelas normales no se aceptaba en los reglamentos de la mayoría de las carreras universitarias, sobre todo en las que gozaban de mayor prestigio social como medicina, abogacía o ingeniería. Sin embargo, las políticas de admisión variaron en las distintas Facultades y carreras universitarias, y en general las nuevas instituciones fueron más permeables al acceso de las mujeres. Destaquemos que en la Argentina, la formación universitaria de las mujeres no contó con programas o instituciones propias y separadas de la enseñanza para los varones tal como aconteció en Estados Unidos, Inglaterra u otros países. ${ }^{2}$ De hecho, en los estatutos y reglamentos universitarios no existieron restricciones formales para que las mujeres pudieran inscribirse como estudiantes en la universidad y obtener un diploma, aunque esto se veía limitado por factores ideológicossociales sobre la "naturaleza" femenina y su papel en la sociedad,

1 En 1924, por ejemplo, los colegios nacionales sumaban 43 establecimientos y contaban con 14.238 alumnos de los cuales el 15\% eran mujeres (la mayoría representaba a las alumnas de los pocos colegios de señoritas existentes). Una proporción similar se mantenía entre los profesores (85\%) y profesoras (15\%) del cuerpo docente integrado por 1.890 profesores. En cambio, la distribución por sexo entre los alumnos inscriptos ese mismo año en las 84 escuelas normales, acusaba: $84 \%$ de mujeres y $16 \%$ de varones de un total de 13.469 estudiantes. A su vez el cuerpo de profesores se componía de 2.155 docentes, donde un $58 \%$ eran mujeres y un $42 \%$, varones. Cabe señalar que en esa época se observaba un incremento constante en el empleo de mujeres en la docencia secundaria. En el caso de los colegios nacionales, en cuatro años se había casi duplicado la proporción de mujeres, ya que en 1920 el profesorado se componía de un $92 \%$ de varones y un $8 \%$ de mujeres. Cierta tendencia hacia la feminización de la actividad docente en la enseñanza secundaria es analizado en Pinkasz, 1992:59. 82.

2 En cuanto a la formación científica de mujeres en Norteamérica, véase, entre otros, Rossiter, 1982; y para el contexto inglés, Richmond, 1997:422-455. En el caso de Uruguay, donde existió una relación muy estrecha entre los movimientos feministas de ambas márgenes del Río de la Plata, el movimiento local de mujeres presionó para el establecimiento de su propia Universidad de Mujeres, creada en Montevideo hacia 1911. 
así como las escasas perspectivas de ejercicio profesional más allá de la docencia en los niveles inferiores del sistema educativo. Esto comenzaría a revertirse lentamente en los inicios del siglo XX y a ello se agregaría la creación de dos colegios secundarios estatales de mujeres en 1907: el Liceo de Señoritas de la Capital Federal y el Colegio de Señoritas de la Universidad Nacional de La Plata, donde se ofrecería la formación requerida para los estudios universitarios y actuarían como docentes las primeras graduadas universitarias en diferentes disciplinas.

Las profesiones relacionadas con las ciencias de la salud fueron las primeras carreras universitarias elegidas por las mujeres en la Argentina. Inicialmente pudieron inscribirse y obtener diplomas en las carreras más cortas y con menor estatus académico: Obstetricia, considerada en general como una profesión femenina derivada de la figura de la "comadrona"; luego Farmacia y Odontología, donde algunas ejercieron en el marco de un emprendimiento familiar o en consultorios privados; $y$ finalmente en los estudios superiores de medicina. Como señala Palermo, varias de las jóvenes que se inclinaron por Farmacia u Odontología compartieron con sus padres, hermanos y maridos el ejercicio profesional, lo que seguramente fue un apoyo para ellas. En 1885 se registra una de las primeras egresadas de Farmacia en la Universidad de Buenos Aires, Elida Passo, hija de un farmacéutico. Anteriormente había ingresado y cursado tres años en la Facultad de Humanidades y Filosofía de esta Universidad, rindiendo también algunas materias en la Facultad de Ciencias Exactas, Físicas y Naturales. Luego de graduarse intentó matricularse en Medicina, donde se le negó inicialmente la inscripción y debió apelar a un recurso judicial para ser admitida, acción que tuvo repercusión en los ámbitos académicos y los diarios de la época. Sin embargo, no pudo completar sus estudios médicos, ya que falleció de tuberculosis antes de recibirse. (Palermo, 2005:59-79) La primera mujer en terminar el doctorado en medicina y cirugía fue Cecilia Grierson (1859-1934) en 1889, quien se había inscripto en la misma Facultad poco después de 
Ni solas ni resignadas

Passo. Es la primera egresada en una carrera superior universitaria que se registra en la Argentina y constituyó un referente importante para otras mujeres que siguieron los estudios de medicina. Como ha sido ampliamente analizado, Grierson tuvo una destacada actuación pública organizando distintas instituciones y colaborando en el movimiento de mujeres en los inicios de siglo XX. (Véase, entre otros, Kohn Loncarica, 1976; Barrancos, 2002) Entre ello participó en la creación y dirección inicial de Consejo Nacional de Mujeres, del cual se separa en 1910; y en la fundación de la Asociación Universitarias Argentinas, donde también participaría la segunda médica diplomada en Buenos Aires en 1892: Elvira Rawson, junto a otras profesionales médicas recibidas en el extranjero y luego otras graduadas universitarias. El desempeño profesional de esas primeras médicas se orientó principalmente hacia la pediatría, la higiene, la "clínica de señoras" y los primeros auxilios, y al mismo tiempo mantuvieron un acercamiento con el feminismo en sus distintas orientaciones, así como con el pensamiento liberal y laicista de la época. (Kohn Loncarica y Sánchez, 1996:113-138)

En la primera década del siglo se diplomaron once médicas en el país, a las que se sumaron dos más formadas en el extranjero, constituyendo menos del $1 \%$ del total de médicos diplomados en ese período. Algunas se habían graduado previamente en Farmacia y Obstetricia, y varias contaron con diplomas de bachiller otorgados por los colegios nacionales. En cuanto a su actuación profesional, ejercieron en consultorios privados y en instituciones públicas vinculadas a la asistencia pública, hospitales, el Departamento Nacional de Higiene, o el Consejo Nacional de Educación. Algunas trabajaron en la educación secundaria y en la enseñanza de enfermeras y parteras, mientras que las que alcanzaron la docencia universitaria, lo hicieron como auxiliares, es decir en los escalafones más bajos de la jerarquía académica. Aunque se presentaron y apelaron para acceder a adscripciones y el acceso como profesoras suplentes en la universidad, esto les fue denegado hasta la década de 1920. En 
esa década, como analizan Kohn Loncarica y Sánchez, se incrementa sensiblemente el número de mujeres diplomadas en medicina en la Universidad de Buenos Aires, entre las que se encuentran las primeras que llegan a desempeñarse como jefas de un servicio sala hospitalaria, una posición médica importante, asî como en el profesorado universitario ${ }^{3}$ y también algunas que realizan una obra científica de cierta importancia. En este grupo, se registra una ampliación de los temas tratados en las tesis doctorales y de las orientaciones profesionales, así como un alejamiento del feminismo "duro", mientras se aplacan las polémicas por la incorporación de la mujer a la profesión médica suscitadas al iniciarse el siglo. (Kohn Loncarica y Sánchez, 1996, 2000)

En la primera década del siglo $\mathrm{XX}$ se incrementa sensiblemente el número de mujeres que siguen estudios universitarios y la presencia femenina se registra en otras carreras y Facultades, así como ocupando nuevos nichos laborales y una ampliación de las esferas de desempeño profesional. El área de las humanidades y las ciencias de la educación, disciplinas vinculadas a los profesorados, fue otro espacio donde se concentró la matriculación femenina en el contexto universitario en los inicios del siglo. En 1901 presentaron sus tesis doctorales el primer grupo de egresados de la Facultad de Filosofía y Letras de la Universidad de Buenos Aires, creada en 1896. De esas primeras nueve tesis,

3 María Faulin, fue la primera egresada que alcanzó el rango de profesora titular ("catedrática") hacia 1923 y lo hizo en una universidad nueva en el interior del país y en una carrera de menor jerarquía académica, donde tradicionalmente tuvieron acceso las mujeres: en la Escuela de Farmacia y ramos menores de Rosario, dependiente de la Facultad de Ciencias Médicas de la Universidad Nacional del Litoral. Este nombramiento de una mujer en una universidad del interior del país, como han señalado Kohn Loncarica y Sánchez, pareció pasar inadvertido, mientras que el nombramiento posterior de María Teresa Ferrari de Gaudino, miembro de una familia tradicional, como profesora adjunta en la cátedra de Obstetricia en la Facultad de Medicina de Buenos Aires en 1927 fue unánimemente celebrado como la primera profesora universitaria argentina. Kohn Loncarica y Sánchez, 2000:89-107. 
Ni solas ni resignadas

cuatro correspondieron a "señoritas" y entre las tesis que obtuvieron calificación de sobresaliente se destacaron la de Ernestina López sobre literatura americana, y la de su hermana Elvira V. López sobre el movimiento feminista de la Argentina. En los siguientes años algunas mujeres presentaron tesis históricas y ensayos sociológicos, y entre ellos, uno de antropología física. En 1911, Juliane A. Dillenius (1884-1949) presentó su tesis sobre craneometría comparativa de los antiguos habitantes de Tilcara, una localidad del Noroeste argentino. Este trabajo fue precedido por dos publicaciones y una comunicación en el XVII Congreso Internacional de Americanistas reunido en Buenos Aires en 1910, siendo una de las tres únicas mujeres que expusieron en ese evento, y en el cual también participó como adherente su padre. (Barrancos, 2000:127-144) Como estudiante de la Facultad de Filosofía y Letras pudo disponer de las colecciones del Museo Etnográfico dependiente de esta institución así como las reunidas por el director del mismo y sus colaboradores en las expediciones por el norte del país. Sus investigaciones contaron con el apoyo y la dirección del profesor de antropología, el alemán Roberto Lehmann-Nitsche (1872-1938), con quien luego se casaría y tendría cinco hijos. A partir de ese momento, al igual que las esposas de otros antropólogos y algunos científicos de la época, se convirtió en colaboradora de los trabajos de su marido.

En la Facultad de Filosofía y Letras, la aceptación de las egresadas de las escuelas normales así como los cursos para el profesorado de enseñanza secundaria implicó una presencia importante de las mujeres de distintas sectores sociales, tanto como alumnas regulares como integrando el auditorio de las conferencias públicas. De hecho, los trabajos doctorales presentados por las egresadas de esta institución constituyeron el 43,24\% de las 37 tesis presentados entre 1901 y 1919 (Candioti, 1920), mientras que en 1923, por ejemplo, el estudiantado femenino representaba más del $51 \%$ de los estudiantes inscriptos. En las restantes Facultades que existían en la Universidad porteña recién después de 1910 aparecen las primeras egresadas en 
abogacía, ciencias naturales, química e ingeniería civil, mientras que en ciencias agronómicas las primeras alumnas se registran en la década de $1920^{4}$ y en otras carreras las mujeres egresan un tiempo después. En la Universidad de Córdoba, se registraba en 1924, 133 alumnas (9\% del estudiantado), la mayoría en la Facultad de Ciencias Médicas, seis mujeres en la de Derecho, tres en la de Ciencias Exactas y Naturales. En la Universidad de Tucumán, creada en 1914 y orientada hacia estudios más técnicos y populares, las mujeres se concentraron principalmente en la carrera corta de farmacia. En el Litoral, la mayor inscripción femenina se daba en las Facultades de Medicina y Ciencias de la Educación y unas pocas alumnas seguían los estudios de abogacía y ciencias económicas.

En la ciudad de La Plata, la concurrencia femenina a las aulas universitarias se detecta desde la creación de la universidad provincial en 1897. La Escuela de Obstetricia y la carrera de Farmacia contaron con alumnas regulares y también algunas mujeres acompañaron a sus esposos a las clases de ciencias jurídicas en calidad de "oyentes". Inicialmente los requisitos de admisibilidad no fueron tan estrictos como en las universidades nacionales de Buenos Aires y Córdoba. Para todos los estudios se permitió la asistencia de oyentes y alumnos libres y además, en la Facultad de Química y Farmacia se aceptaron inicialmente como estudiantes regulares a los egresados de las escuelas normales, lo que facilitó el ingreso de mujeres. El desempeño de las primeras alumnas al iniciarse los cursos de química y farmacia llamaron la atención del fundador y rector de la Universidad, Dardo Rocha, quien en una carta al senador provincial Rafael Hernández, dando

4 En 1924, se registra una alumna inscripta en la Facultad de Agronomía y Veterinaria de Buenos Aires sobre un total de 285 estudiantes. En la Facultad de Ciencias Económicas se encuentran cuatro mujeres entre 422 estudiantes, mientras que en Filosofía y Letras, la concurrencia femenina alcanza el 56 \%. En los registros de las otras Facultades, no se presenta información discriminada por sexo. Memoria presentada al Congreso Nacional por el Ministro de Justicia e Instrucción Pública. Buenos Aires, Talleres de la Penitenciaria Nacional, 1926. 
Ni solas ni resignadas

cuenta del primer año de funcionamiento de la institución, señalaba:

en la Facultad de Química existen una señora y dos niñas que habían obtenido las más altas calificaciones entre sus compañeros: es pues la iniciativa de una nueva carrera para la mujer, problema tan interesante de la civilización moderna... ${ }^{5}$

Dos de estas primeras alumnas, ya que una falleció antes, se graduarían de farmacéuticas en 1901, luego otras tres mujeres egresarían al año siguiente ${ }^{6}$ y algunas más continuarían esos estudios en los siguientes años. Especialmente la profesora norteamericana Mary O'Graham, directora de la Escuela Normal Nacional de La Plata, promovió que sus alumnas continuaran estudiando en la Universidad y también por sus clases pasaron algunas destacadas feministas de la primera década del siglo XX, como la militante socialista Raquel Camaña y la uruguaya María Abella Ramírez. En esta ciudad, creada en 1882 para servir de capital a la Provincia de Buenos Aires, en la transición del siglo existió un importante movimiento librepensador y sectores laicistas favorables a la educación de las mujeres y su participación en la

5 Archivo General de la Nación, Fondo Dardo Rocha, Leg. n 2958.

${ }^{6}$ Las primeras dos egresadas fueron: Celia Zeballos de Heredia, quien había estudiado en la Escuela Normal Nacional de la Capital Federal y mientras cursaba dirigía una escuela pública. Luego continuaría estudiando en la Sección Pedagógica de la Universidad de La Plata, llegando a dirigir el Colegio de Secundario de Señoritas de esta universidad. Isabel Baldevidares, una de las primeras egresadas de la Escuela Normal de Profesoras de La Plata, desde 1884 ejercía la docencia y en 1890 había ingresado a la Escuela Normal como profesora, cargo al que renunció cuando comenzó a estudiar en la Facultad. Esta última inició sus estudios universitarios junto a su hermana, quien falleció en 1898. En 1902 se recibieron: María Luisa Rocha, Mercedes Mouzo Oliver y María Luisa Gutiérrez, las tres habían egresado de la Escuela Normal de La Plata en 1897 e ingresado a la Facultad al año siguiente. Posteriormente Gutiérrez continuarían estudiando ciencias naturales y química en el Museo/Facultad de Ciencias Naturales de La Plata. 
vida pública. En ese sentido, por ejemplo, se puede mencionar la presencia de mujeres como conferencistas en la Biblioteca Pública al iniciarse el siglo XX y luego en los ciclos de extensión universitaria, donde se escucharon diferentes voces en torno al debate sobre la capacidad y "naturaleza" de la mujer; así como la creación de algunas revistas feministas vinculadas con la Liga Nacional de Mujeres Librepensadoras y su programa de defensa de los derechos civiles y políticos de las mujeres. En este contexto se graduó en 1909 la primera abogada argentina, María A. Barreda, quien contó con una campaña a su favor para ser aceptada en la Corte Suprema provincial y poder ejercer su profesión. Esto sentó precedente para, poco después, defender el derecho de la mujer argentina el ejercicio de la profesión de contadora pública, tal como se había aceptado en el caso de una extranjera nacionalizada.

La mujeres fueron admitidas en las distintas Facultades y carreras que se organizaron al establecerse la Universidad Nacional de La Plata en 1905, sobre la base de un conjunto de instituciones que venían funcionando bajo el gobierno provincial, como la antigua Universidad, la Facultad de Agronomía y Veterinaria, el Museo General de La Plata, la Biblioteca Pública, el Observatorio Astronómico, y el Colegio Nacional. En la Sección Pedagógica, destinada a la formación de profesores secundarios, se admitió la inscripción de maestros normales, con clasificación sobresaliente en el último curso y la aprobación de algunas materias de quinto año del colegio secundario. En este sentido, los estudios pedagógicos constituyeron una vía importante de acceso a la universidad para las mujeres y sectores de orígenes sociales más modestos y de distintas localidades de la provincia de Buenos Aires y del interior del país. En este nuevo contexto universitario se abrirían para las mujeres varias posibilidades de estudio y también de inserción laboral en el ámbito académico. Entre ello, mencionemos la incorporación de una mujer extranjera como profesora adjunta en el Instituto de Física en 1909, época en que en otras instituciones universitarias las mujeres argentinas que se 
Ni solas ni resignadas

presentaban para ser profesoras suplentes eran rechazadas. La incorporación de la físico-química danesa Margrete Heiberg de Bose (1865-1952) a cargo de un curso práctico de física experimental fue parte de las condiciones de trabajo exigidas por su marido, Emil Bose (1874-1911), quien fue contratado para la reorganización de los estudios en ciencias físicas y para promover las investigaciones científicas. ${ }^{7}$ De hecho, Emil Bose firmó el contrato de trabajo de su esposa, ya que de acuerdo a las costumbres y la legislación vigente hasta 1926 una mujer casada no disponía de derechos civiles para administrar bienes ni firmar contratos de trabajo. Tras la muerte de su marido, Margrete Bose siguió en ese cargo un tiempo más, luego viajó a Europa y después de la primera guerra mundial se instaló de nuevo en la Argentina, incorporándose nuevamente en el profesorado del Instituto de Física, como profesora extraordinaria, hasta su jubilación. Cabe señalar que esta presencia femenina no parece haber despertado el interés de otras mujeres por investigaciones o los estudios en ciencias físicas en esta institución, donde, por otro lado, muy contados alumnos siguieron esta carrera en las primeras décadas del siglo.

Por el sistema de correlación de estudios de la Universidad de La Plata, los estudiantes del profesorado debían asistir a otras Facultades y dependencias para completar la formación especial y científica de las asignaturas a enseñar, mientras que los alumnos de las distintas carreras universitarias podían optar por los cursos pedagógicos para obtener también el título de profesor. La obtención del título profesional junto con el de profesor, que ofrecía mayores posibilidades laborales, fue una situación frecuente entre las diplomadas en farmacia, química, ciencias naturales y las primeras ingenieras agrónomas. Hacia 1912 se

${ }^{7}$ Margrete Heiberg fue la primera mujer en recibir el Magister Scientiarum en química otorgado por la Universidad de Copehagen, contactándose con destacados investigadores de la época y completando su entrenamiento en Göttingen, donde conoció a su futuro marido. Su carrera ha sido analizada en Pyenson, 1985 y von Reichenbach, 2005. 
graduaron en la Facultad de Agronomía las primeras ingenieras agrónomas de país, dos de las cuales también obtuvieron el título de profesoras. ${ }^{8}$ Una de ellas, Cecilia Silva Lynch, estudió además ciencias naturales en el Museo de La Plata, donde en 1911 obtuvo una beca para dedicarse a esos estudios. Completó la licenciatura en ciencias naturales y había elegido investigar sobre la embriología del escuerzo como tema de tesis doctoral, aunque no llegó a terminar ni publicar nada sobre esto. Ese tema estaba relacionado con las investigaciones que se promovían en el laboratorio de Zoología del Museo, donde los dos profesores de esta materia, ensayaron por varios años, y sin éxito, la cría y reproducción en cautiverio de algunas especies de esos animales.

Cabe destacar, el alto porcentaje de alumnas que se interesaron por las distintas carreras que dependían del Museo de La Plata. Esta institución creada en 1884 fue incorporada a la estructura de la Universidad Nacional de La Plata en 1906, como Instituto de Investigaciones y Facultad de Ciencias Naturales. Dentro de esta unidad académica se organizaron las carreras de farmacia, los doctorados en ciencias naturales y en química, y los profesorados en geografía y en dibujo. Todos estos estudios contaron desde sus inicios con la inscripción de mujeres. De hecho, las estadísticas de alumnos discriminadas por sexo en 1917, muestran que ese año la matriculación femenina alcanzó el 38\% del total de estudiantes en las distintas carreras de esta Facultad. (Lafone Quevedo, 1918:106-122) Como se mencionó anteriormente, la concurrencia femenina en los estudios de farmacia se manifestaba desde la época de la Universidad provincial, y algunas de ellas buscaron luego continuar sus estudios en ciencias naturales o el doctorado en química. En otros casos, la

\footnotetext{
8 Las primeras agrónomas diplomadas fueron: Ninfa Fleury de Escalada, Cecilia Silva Lynch y Amalia Vicentini, quien presentó una tesis sobre la enseñanza de la agronomía. Las dos últimas también recibieron el diploma de profesor de enseñanza secundaria y superior. Facultad de Agronomía, UNLP. Nómina de titulados y egresados desde la fundación de la Facultad en 1883 hasta el 31 de diciembre de 1925. La Plata, 1926. Véase también: Graciano, 1998:13-72.
} 
Ni solas ni resignadas

matriculación en las carreras científicas fue posible para aquellas que contaban con el título de bachiller del Colegio Nacional de La Plata, que inicialmente había recibido algunas alumnas, especialmente a las hijas de ciertos profesores y de la élite local. En 1907, cuando este Colegio se incorporó a la Universidad, la gran inscripción de alumnas hizo que las autoridades de la Sección Pedagógica propusieran separar la enseñanza de las mujeres, creándose para ellas el Colegio Secundario de Señoritas, donde se otorgaba el diploma de bachiller para ingresar a la universidad, ofreciendo, al mismo tiempo, un campo laboral para las que siguieron estudios superiores. Otras, especialmente aquellas que disponían del título de maestras pero no de bachiller, ingresaron a la Facultad de Ciencias Naturales tras haber realizado estudios en la Sección Pedagógica. De hecho, casi todas las que siguieron la licenciatura o el doctorado en ciencias naturales, realizaron previamente o de forma paralela los cursos del profesorado, carrera que ofrecía mayores posibilidades laborales. En 1907, se permitió la inscripción de maestras normales en la Escuela de Geografía y posteriormente en la carrera de Farmacia, lo cual generó un aumento de la matrícula estudiantil. Una tercera vía de acceso a los estudios universitarios, se abrió en la década de 1910 cuando la Facultad de Agronomía y Veterinaria aceptó, tras aprobar un examen de ingreso, la matriculación como alumnos regulares a personas que no tenían certificado de estudios secundarios. Por esa vía, algunas alumnas - y también varones que fueron admitidos como regulares en aquella Facultad, pidieron el traspaso y su reconocimiento como estudiantes universitarios para ingresar en la Facultad de Ciencias Naturales.

En el caso de los estudios en ciencias naturales, la presencia femenina se registró desde los primeros cursos que se ofrecieron en el Museo/Facultad a partir 1906, donde hasta la década de 1930 fueron muy pocos los alumnos que se inclinaron por estos estudios como también sucedió en los otros dos centros de formación en estas disciplinas que existían en el país. El entrenamiento formal universitario en ciencias naturales se 
consolidó recién en los inicios del siglo XX. Si bien desde el último tercio del siglo XIX se había establecido en las Universidades de Buenos Aires y Córdoba planes de estudio y diplomas que acreditaban competencia en estas disciplinas, esta carrera prácticamente no había contado con estudiantes y los diplomas otorgados habían consistido principalmente en los títulos revalidados por los propios profesores extranjeros $y$ reconocimientos honoríficos. En Córdoba, un solo alumno completaría el Doctorado en Ciencias Naturales en el siglo XIX y posteriormente la carrera dejó de existir hasta su reorganización en la década de 1920, y en esta época fue una alumna formada en La Plata la primera en obtener nuevamente ese diploma. En la Facultad de Ciencias Exactas, Físicas y Naturales de la Universidad de Buenos Aires, la Escuela de Ciencias Naturales comenzó a tener alumnos regularmente al iniciarse el siglo XX y en 1902 se registra el primer diplomado. En la primera década los alumnos matriculados anualmente en esta institución al igual que en La Plata, no llegaban a superar la decena, incrementándose lentamente su número en los siguientes años.

En el contexto del Museo/Facultad de Ciencias Naturales de La Plata, varones y mujeres se formaron en ciencias naturales en estrecha interacción desde la organización de esta carrera, participando en los mismos circuitos de formación y de sociabilidad estudiantil. Entre esto último, se puede mencionar la participación de las mujeres como socias y también en la comisión directiva - algo poco usual en la época - del Centro de Estudiantes del Museo creado en 1906, desde donde se organizó una revista, excursiones, conferencias de repaso, reuniones periódicas y otros eventos sociales. Entre 1906 y 1930, la inscripción de mujeres en ciencias naturales en La Plata alcanzó el $48 \%$ de los 46 estudiantes regulares y una proporción similar se mantuvo entre los 18 alumnos que se doctoraron entre 1912 y 1936. (García, 2004) De hecho, el primer alumno en terminar la licenciatura en ciencias naturales, junto con el profesorado, fue Carolina Etile Spegazzini (1887-1925) en 1910, quien además 
Ni solas ni resignadas

estudió paralelamente farmacia y completaría el doctorado en química en 1918, pero fallecería antes de terminar su trabajo de tesis doctoral en ciencias naturales. Hija mayor y colaboradora del profesor de botánica, el italiano Carlos Spegazzini, había cursado sus estudios secundarios en el Colegio Nacional de La Plata y junto con otras compañeras ingresó en 1906 a la nueva Facultad de Ciencias Naturales. Allí fue la primera alumna en ser nombrada ayudante en 1907, luego también se le otorgaría una beca de estudios y tras doctorarse fue ascendida a jefe de trabajos, desempeñándose luego en ese cargo en la nueva Facultad de Química y Farmacia de la Universidad platense hasta su muerte. Asimismo fue docente en una escuela normal de maestras y estuvo vinculada al Instituto Bacteriológico Nacional $y$ al Laboratorio de Bacteriología del Hospital Italiano de Buenos Aires. (Toffoli de Mateos, 1987) En 1911 publicó en la Revista del Museo de La Plata su primer trabajo de investigación, realizado dos años antes junto con una compañera de estudios, María Luisa Cobanera, sobre análisis de la grasa de camello, obtenida de los restos de un animal muerto en el Jardín Zoológico local y que fue remitido para las colecciones del Museo. Ese mismo año también publicó otro trabajo en la Revista de la Universidad de Buenos Aires sobre las propiedades curativas de una planta usada popularmente, realizado en coautoría con el vicedirector del Museo de La Plata y Jefe de la Escuela de Farmacia y Química de ese instituto, el químico Enrique Herrero Ducloux. Este profesor también le sugeriría el tema, le facilitaría datos y dirigiría su tesis doctoral en química, para lo cual también contó con el acceso a la biblioteca particular del químico Pedro N. Arata, facilitado seguramente por sus vínculos familiares y académicos. ${ }^{9}$ Asimismo,

9 La tesis de Carolina Spegazzini consistió en un estudio crítico del método de Carnot para la determinación de las sales de potasio. Herrero Ducloux le había sugerido el tema, con el objeto de poder observar y catalogar las anomalías que encontraban él y sus ayudantes en el Laboratorio del Ministerio de Agricultura de la Nación al aplicar este método en algunos casos. Toffoli de Mateos y Spegazzini, 1987. 
Herrero Ducloux dirigió los estudios experimentales realizados por María Luisa Cobanera ${ }^{10}$ en los laboratorios químicos del Museo, sobre la acción de las sales de cobalto y vanadio en vegetales, cuyos resultados se publicarían en la Revista del Museo de La Plata y luego serían ampliados en su tesis doctoral presentada hacia 1913.

En la Escuela de Ciencias Naturales de la Universidad de Buenos Aires, un cierto aumento en la matriculación general se debió a la concurrencia femenina, doctorándose las primeras en 1912: Juana Guillermina Dieckmann (1888-1960), Ana y Lía Acevedo. Las tres habían sido alumnas del botánico Cristóbal Hicken en el Colegio Nacional central, quien también se desempeñaba como docente en la Facultad de Ciencias Exactas, Físicas y Naturales de Buenos Aires y apadrinó el trabajo de tesis doctoral de estas tres alumnas. La primera recibió la calificación de sobresaliente y las facilitaciones del jurado por su trabajo de tesis doctoral, junto con el premio Stroebel, un reconocimiento que entregaba la Facultad al mejor egresado en estas disciplinas. Fue una de las primeras socias de la Sociedad Argentina de Ciencias Naturales, creada en 1911 y participó en la organización de la Primera Reunión Nacional de Ciencias Naturales realizada en Tucumán en 1916, a la que asistió junto con su marido. Se dedicó a la enseñanza de Historia Natural en establecimientos de educación media, escribió algunos trabajos botánicos,

\footnotetext{
${ }^{10}$ María Luisa Cobanera había cursado sus estudios en el Colegio Nacional de La Plata y luego se recibió de farmacéutica junto con Carolina Spegazzini. Entre 1911 y 1912 contó con una de las becas del gobierno para perfeccionar sus estudios. En esa época participó como conferenciante en un ciclo de divulgación organizado en el anfiteatro del Museo, a cargo de los primeros egresados y estudiantes avanzados de química y farmacia. Cabe señalar que fue la única alumna que dictó una conferencia en ese ciclo, una actividad en la cual generalmente las estudiantes sólo participaban en calidad de oyentes. Fue la segunda mujer en terminar el doctorado en la Escuela de Química y Farmacia platense y su director tenía expectativas de que pudiera desarrollar un carrera científica, pero poco después de terminar sus estudios se casó y mudó a la Provincia de Córdoba.
} 
Ni solas ni resignadas

traducciones del alemán y artículos de divulgación científica en periódicos, estos últimos firmados con el seudónimo de "Nepenthes". (Hicken, 1923)

En la década de 1910 la presencia de alumnas en los estudios de ciencias naturales y química se registra todos los años. La poca concurrencia de alumnos en ciencias naturales pareció ser un factor que favoreció especialmente la situación de las mujeres que se inclinaron por estos estudios. De hecho, en este campo las alumnas obtuvieron becas para realizar sus estudios universitarios, recibieron distinciones y premios por sus trabajos doctorales y varias serían convocadas para desempeñarse como colaboradoras y ayudantes en secciones de las oficinas de investigación del gobierno $y$ en museos. Los cargos auxiliares así como las "adcripciones" ad-horem (permisos especiales para trabajar en determinada sección y encargarse del estudio de una colección o determinado grupo de especimenes) que se generalizaron en el Museo Nacional de Buenos Aires a partir de 1920 y en otros centros científicos, constituyeron reconocimientos institucionales que posibilitaban no solo acceder a colecciones, material de estudio y bibliografía especializada sino también publicar en las revistas y otras ediciones oficiales de estas instituciones, desde donde se difundía el trabajo de su personal y los estudios realizados a partir de sus colecciones. La edición y distribución de publicaciones permitía a las instituciones, pero también a su personal, ingresar en los circuitos institucionales de canje de bibliografía y a distintas redes de intercambio y debate en el ámbito local e internacional. En ese sentido, una publicación institucional situaba el trabajo del científico en otro marco de legitimidad y circulación. Las mujeres argentinas comenzaron a participar en este marco al acceder a una educación científica superior y contactos académicos que posibilitaron su incorporación en los espacios institucionales de trabajo científico. 


\section{Empleo y espacios de trabajo científico para las naturalistas}

En la primera década del siglo XX, algunas instituciones científicas argentinas comienzan a contar con alguna mujer entre su personal técnico. Entre las primeras empleadas que se registran, algunas eran de origen extranjero y varias formaban parte del entorno familiar de investigadores o técnicos de esos establecimientos. Así, por ejemplo, en 1906 la lista de personal del Observatorio Astronómico de Córdoba, integrado principalmente por norteamericanos, incluye dos mujeres, Violeta E. Barter y Hilda G. Symonds ${ }^{11}$, cuyos apellidos son los mismos de otros integrantes. Sus tareas aparecían vinculadas a mediciones y cómputos, una actividad que remite al "trabajo de mujeres" en ciencia señalado por Margaret Rossiter. (Rossiter, 1980 e 1982) En el Museo Nacional de Historia Natural de Buenos Aires, junto con el botánico belga Lucien Hauman, Germainne Vanderveken se desempeñó unos años como conservadora del herbario y fue coautora del primer volumen del catálogo de fanerógamas argentinas, publicado en 1917. (Parodi, 1961) En la Sección de Zoología aplicada del Ministerio de Agricultura de La Nación, el zoólogo francés Fernando Lahille incorporó entre sus ayudantes a la profesora Teresa Joan, presumimos de origen extranjero, quien colaboró en la confección de estadísticas de pesca, la identificación de enemigos naturales de ciertas plagas y el estudio de parásitos y otros invertebrados por varias décadas. Se especializó en helmintología, firmando algunos trabajos junto a su director, pero en su mayoría sola. Sus principales tareas se realizaron en torno al espacio del gabinete y el laboratorio, estudiando ejemplares infectados y especimenes que le enviaban para su determinación, ya que a diferencia de los otros ayudantes varones de esa Sección, no hay registros que indiquen que ella fue

\footnotetext{
${ }^{11}$ Expediente n 33 "Observatorio de Córdoba”, Caja 5, año 1906, ContaduríaMinisterio de Justicia e Instrucción Pública. Archivo General de La Nación (Sección Intermedio)
} 
Ni solas ni resignadas

enviada en misión oficial para hacer trabajo de "campo" por el interior del país.

En el Museo de La Plata se abrieron posibilidades laborales para las mujeres a partir de su transformación en Facultad y la creación de cargos rentados de ayudantes alumnos, con los que las autoridades universitarias buscaron incentivar el entrenamiento en tareas científicas-técnicas y ayudar económicamente a los estudiantes. (García, 2004c) Las reglamentaciones de estos cargos fueron muy estrictas con respecto a las altas calificaciones que debían poseer los candidatos y el mantenimiento de los mismos estaba supeditado a la aprobación regular de materias sin aplazos. Estos empleos y especialmente las becas de estudios que se consiguieron a partir de 1910 constituyeron un incentivo para reclutar los pocos alumnos que se interesaron por los estudios de ciencias naturales. Según las cátedras y secciones, los cargos de ayudantes fueron destinados para ayudar en el desarrollo de las clases prácticas, colaborar en la preparación de material didáctico, auxiliar en el cuidado de colecciones o en las actividades de investigación de los profesores. Los alumnos constituían una fuerza de trabajo calificada y de bajo costo para auxiliar en las tareas cotidianas de los laboratorios y gabinetes. Para los estudiantes y jóvenes graduados, los puestos de ayudantes y jefes de laboratorio facilitaban el acceso y el uso de las instalaciones, equipos, colecciones y otros materiales para realizar trabajos científicos. Asimismo, esas posiciones abrían oportunidades de entrenamiento y de entrelazar los primeros pasos para desarrollar una carrera académica, que continuaba con el ascenso como jefes de trabajos prácticos o de laboratorio, y en caso de los varones, con los nombramientos como profesor suplente o adjunto y luego titular. Las egresadas en ciencias naturales, en cambio, recién en la década de 1930 pudieron alcanzar estos últimos puestos.

$\mathrm{El}$ acceso de las alumnas a los cargos de ayudantes y luego a las becas de estudio en el Museo de La Plata se vió facilitado por los pocos estudiantes que seguían de forma regular estos estudios y la exigencia de no desaprobar ningún examen. En general, las 
alumnas de ciencias naturales se destacaron por ser mejores estudiantes y tener altas calificaciones. Como mencionamos anteriormente, la primera en ser nombrada ayudante fue Carolina Spegazzini en 1907 y tras doctorarse fue ascendida a jefe de trabajos prácticos. Al igual que ella, la mayoría de los alumnos y egresados que siguieron regularmente los estudios de ciencias naturales en el Museo tuvieron acceso a un cargo de auxiliar y/o a alguna de las becas del gobierno que se consiguieron entre 1910 y 1914, para estimular "las aptitudes y el entusiasmo" de aquellos alumnos que hubieran demostrado vocación por profundizar su cultura científica. El importe de estas becas era de 150 pesos mensuales, igual que la remuneración de un Jefe de Laboratorio o de trabajos prácticos y el doble que la que percibía los ayudantes alumnos o un maestro de escuela. La posibilidad de acceder a estas becas pareció estimular la inscripción de algunos alumnos y alumnas en la carrera de ciencias naturales, quienes al mismo tiempo solicitaban la admisión en la Facultad y una beca de estudio. Con algunas excepciones, varios de estos casos muestran que los estudiantes de ciencias naturales no provenían de sectores privilegiados o altos ingresos. En relación con ello, podemos mencionar por ejemplo, la trayectoria de Ana Manganaro (18911921), nacida en La Plata e hija de modestos inmigrantes italianos. Desde los cinco años frecuentó las escuelas estatales y luego la Escuela Italiana, en la que obtuvo medalla de oro por sus estudios, y en la Escuela Normal local, donde se recibió de maestra en 1910. En esta época comenzó a interesarse por los estudios botánicos y a confeccionar un herbario con ejemplares reunidos en la localidad platense y en otros pueblos de la provincia que tuvo ocasión de visitar. Cabe señalar, que las herborizaciones y observaciones por el bosque que rodeaba el Museo era una práctica muy difundida tanto en la enseñanza primaria y media de mujeres como en los cursos universitarios para naturalistas y profesores. En 1911, Manganaro se inscribió en la Facultad de Agronomía y Veterinaria y pocos meses después pidió su pase a la Facultad de Ciencias Naturales, comenzando paralelamente a 
Ni solas ni resignadas

cursar en la Sección Pedagógica de la Universidad platense. En 1913 se recibió de farmacéutica y profesora de enseñanza secundaria en química y ciencias afines y ese mismo año aparece publicado su primer trabajo científico en los Anales de la Sociedad Científica Argentina. Luego comenzó el doctorado en ciencias naturales y obtuvo una de las becas de estudio. Paralelamente se desempeñó como ayudante "ad-honorem" en la Sección Botánica del Museo de esos años. Sus ingresos regulares provenían del trabajo en una farmacia y para mantener a sus hermanos menores, tras la muerte de sus padres, también ejerció de maestra. En 1917 trabajó de forma interina en el Liceo de Señoritas de Buenos Aires y al año siguiente fue nombrada preparadora en el Museo de La Plata, aunque esto quedó en suspenso en el marco del movimiento de reforma universitaria En esa época presentó su tesis sobre la flora leguminosa silvestre de la Provincia. Tras doctorarse fue nombrada Jefe de Trabajos prácticos en botánica en la recién creada Escuela de Medicina de la Universidad de La Plata y poco antes de morir gestionaba un puesto en el Consejo Nacional de Educación. Dejó inéditos varios trabajos, notas y apuntes, uno de los cuales sobre histología de leguminosas fue publicado póstumamente por iniciativa del botánico Carlos Spegazzini.

Los estudios zoológicos en el Museo de La Plata, atrajeron varias alumnas durante la década de 1910 y 1920. Entre ellas se destacó María Isabel Hylton Scott (1889-1990), siendo la primera en doctorarse en ciencias naturales en esta institución. Había nacido en Córdoba, donde cursó en la Escuela Normal, recibiéndose de maestra en 1908. Al año siguiente ejerció como docente en su ciudad natal y luego decidió continuar su formación en la Sección Pedagógica de la Universidad Nacional de La Plata, donde obtuvo el diploma de profesora en Pedagogía y Ciencias afines en 1911. Ese año actuó como prosecretaria en el Colegio de Señoritas, donde seguramente entró en contacto con la socialista y feminista Alicia Moreau, también docente en esa institución y con quien colaboró en la búsqueda bibliográfica de temas vinculados a 
la mujer, las profesiones y la universidad, sustento de artículos publicados en el periódico socialista la Vanguardia. (Castellanos, 1990:3-4) En los siguientes años trabajó como maestra y hacia 1913 comenzó a cursar el doctorado en ciencias naturales en el Instituto del Museo, donde obtuvo una beca de estudios. Al año siguiente fue nombrada ayudante y poco después Jefe de Laboratorio en la cátedra de Zoología, donde comenzaría a realizar sus primeras investigaciones científicas.

El Laboratorio de Zoología del Museo fue un espacio importante de socialización científica, donde los alumnos pudieron disponer de bibliografía especializada, microscopios, micrótomos, elementos de microfotografía y pequeños acuarios para sus trabajos. Allí no sólo se daban clases prácticas como también cursos avanzados en técnicas y prácticas de investigación y posteriormente se organizaron seminarios y reuniones de discusión entre alumnos y exalumnos, entre las que se encontraron varias mujeres. Las actividades en este Laboratorio fueron dirigidas por el profesor Miguel Fernández (1882-1950) (García, 2004b), dedicado a investigaciones embriológicas y anatómicas y que contaba con la colaboración de su esposa alemana Kati Marcinowski (1871- ?) ${ }^{12}$, su compañera de estudios en la Universidad de Zürich. En la Argentina, esta zoóloga trabajó sin ninguna inserción institucional formal, participando en las investigaciones de su marido y desarrollando algunos trabajos propios sobre batracios. Colaboró dibujando cuadros demostrativos para las clases de genética de Fernández,

\footnotetext{
${ }^{12}$ Kati Marcinowski se doctoró dos años después que Fernández, con una tesis sobre la formación del endotelio vascular y la sangre en anfibios, sosteniendo conclusiones similares a las de su futuro marido sobre el origen mesenquimático del sistema vascular y los elementos sanguíneos. Ambos trabajos fueron realizados en el laboratorio del investigador Arnold Lang en la Universidad de Zürich. Participó en las investigaciones de Fernández sobre la embriología de la mulita, describiendo la formación del sistema nervioso. En la década de 1920 publicó algunos trabajos sobre la reproducción y desarrollo embrionario de batracios autóctonos del país, llamando la atención, al igual que otros científicos extranjeros, sobre las características particulares que presentaban la fauna local.
} 
Ni solas ni resignadas

ensayando en la cría y reproducción de animales para estudio y también apoyando las investigaciones de algunas estudiantes de zoología. En ese contexto, Hylton Scott realizó su investigación doctoral y otros trabajos de embriología, entre ellos uno en coautoría con Marcinowski. Asimismo a principios de 1916, Hylton Scott participó en un viaje de estudios a Puerto Madryn, organizado por Fernández para incentivar la enseñanza de la biología marina, el estudio de especimenes vivos de fauna acuática y recolectar diversos organismos. Meses después presentó su tesis sobre el desarrollo embrionario de una especie local de peces vivíparos, realizada bajo dirección Fernández, obteniendo las calificaciones más altas y las recomendaciones del jurado para su publicación. Poco después terminaría su tesis en zoología, su compañero de estudios y el futuro esposo de esta alumna: Maximiliano Birabén. La comparación de los itinerarios iniciales en la carrera académica de este matrimonio, en la que Birabén llegó ejercer en profesorado universitario en la década de 1920, ilustran sobre las diferencias y posibilidades abiertos para mujeres y varones con igual formación y similares antecedentes científicos.

Hylton Scott se desempeñó como Jefe de laboratorio de Zoología hasta que fue separada de su cargo en 1924, continuando luego sus investigaciones en forma privada, reuniendo colecciones y realizando excursiones con su marido y sus hijos, para lo cual equiparon una camioneta-laboratorio. Esta zoóloga fue dejada cesante en el marco de una serie de conflictos entre el Director del Museo, Luis María Torres y un grupo de profesores, entre ellos, Fernández. Posteriormente, este profesor terminó renunciando a la cátedra y la jefatura del Departamento de Biología del Museo. Este cargo vacante fue disputado por los graduados de la institución frente a la contratación de un joven zoólogo alemán, Erich Dautert, doctorado un año antes, y que sólo contaba con un trabajo científico y el entrenamiento en estaciones marinas europeas. Su contratación directa y la ausencia de un concurso público para llenar los cargos fue denunciada por discípulos de Fernández con el respaldado de la Sociedad 
Argentina de Ciencias Naturales y algunos sectores socialistas, insistiendo en que ese joven zoólogo alemán iba a ocupar un puesto para el que las universidades argentinas ya contaban con personal formado. (Podgorny, 1996:60-64) Finalmente, las autoridades universitarias decidieron que se llamaría a concurso de antecedentes para cubrir esos cargos. En ese contexto, se publicó el trabajo doctoral de Hylton Scott en los Anales del Museo Nacional de Buenos Aires, con el respaldo del director de esa institución, Martín Doello Jurado y los comentarios favorables de los zoólogos más importantes del país. Asimismo, ella junto a su marido y otra joven doctora, Ernestina Langmann, insistieron en 1929 para que sus antecedentes científicos y académicos se tuvieran en cuenta para el concurso del cargo de profesor de zoología y Jefe de Departamento en Biología. Sin embargo, como esto último implicaba realizar excursiones y trabajo de campo, el director del Museo, Luis María Torres argumentó que:

\section{no pueden ser incluídas en esta lista porque su condición de mujeres no les permite cumplir con todas las obligaciones inherentes al cargo, tales como salir a expedicionar en los diferentes Territorios de la República. ${ }^{13}$}

El resto de los consejeros académicos del Museo acordaron con esa opinión y se eliminó a las egresadas como candidatas en este concurso, el cual continuó con impugnaciones y problemas. Finalmente, se nombraría a Max Birabén como profesor de Zoología y a otro graduado, Emiliano Mac Donagh como Jefe de Departamento. Tras los cambios políticos-institucionales y en la dirección del Museo, Hylton Scott pudo ganar en 1933 el concurso de profesor suplente de zoología especial, cargo ad-honorem en la misma cátedra de su marido, y luego otros puestos. En esa época

\footnotetext{
${ }^{13}$ Libro de Actas de reuniones extraordinarias del Consejo Académico y Asambleas de Profesores, Folio 55 y 56, Archivo Histórico del Museo de La Plata.
} 
Ni solas ni resignadas

comienza a especializarse en malacología, publicando numerosos trabajos en esta área y recibiendo varias distinciones.

En la década del 1910, algunas alumnas de ciencias naturales comenzarían a ser incorporadas como auxiliares en distintos departamentos de investigación aplicada. En algunos casos, las estudiantes vieron facilitado su ingreso en centros científicos gracias a las destrezas y habilidades que mostraron como dibujantes, un arte que formaba parte de la educación y el ocio de muchas niñas de la época. Si bien inicialmente fueron valoradas como "eficaces colaboradoras" por sus destrezas artísticas y su entrenamiento científico, algunas también demostraron rápidamente su capacidad para desarrollar sus propias investigaciones. Esto se ilustra, por ejemplo, en la trayectoria de Juana Miguela Petrocchi (1893-1925), nacida en Avellaneda, una localidad vecina a la Capital Federal, donde estudió y trabajó. Realizó sus estudios secundarios en el recién inaugurado Liceo Nacional de Señoritas de la Capital Federal, luego frecuentó los cursos del Instituto de Educación Física, del cual egresó como Profesora en 1913. Al terminar sus estudios secundarios fue designada ayudante de laboratorio en una escuela normal de maestras. En 1914 se inscribió en la Facultad de Ciencias Exactas, Físicas y Naturales de la Universidad de Buenos Aires, donde obtuvo la primera beca de estudios para seguir el doctorado en ciencias naturales establecida en esa Facultad, concluyendo sus estudios en 1917. Según sus biógrafos, durante sus años estudiantiles se destacó como una gran dibujante y en 1916 fue contratada para desempeñar esa tarea en el Museo del Instituto Bacteriológico, dependiente del Departamento Nacional de Higiene. Luego fue designada ayudante honoraria de la sección Zoología en esa institución, comenzando a especializarse en el campo de la entomología bajo la dirección de A. Neiva, un especialista brasileño en entomología aplicada. Allí también se contactó con el investigador alemán J. Mühlens, dedicado a enfermedades tropicales. Bajo el estímulo de estos investigadores, Petrocchi se dedicó a la sistemática de mosquitos argentinos y 
especialmente al estudio de los transmisores del paludismo. Asimismo fue ayudante en la cátedra de Higiene de la Facultad de Medicina de Buenos Aires. En 1924 realizó tres viajes, en compañía de Mühlens, al norte argentino para sus investigaciones sobre la dispersión y sistemática de mosquitos, "demostrando que la mujer también puede realizar tareas en la naturaleza" (De Santis, 1992) reuniendo importantes colecciones y observaciones. Al regresar de su último viaje sufrió un ataque de apendicitis hipertóxica, falleciendo poco después. Sus publicaciones entre 1919-1925 sumaron 8 trabajos, la mayoría en la Revista del Instituto Bacteriológico Argentino, y otras ediciones del Departamento Nacional de Higiene, entre ellos una guía para la clasificación de mosquitos trasmisores. Dejó varios otros trabajos sin terminar y la descripción de 11 especies nuevas de mosquitos, de las cuales cuatro se consideraron luego sinónimos (Deautier, 1930-31:271-274; 1925:417-20), y póstumamente recibió varios reconocimientos.

Juana Petrocchi pareció ser bastante apreciada en la época y sus profesores reconocieron su preparación científica. De hecho, en 1920 su profesor de zoología en la Universidad de Buenos Aires, Angel Gallardo, la recomendó como candidata para la cátedra de zoología de la Facultad de Ciencias Exactas y Naturales de la Universidad de Córdoba, donde tras el movimiento reformista se procuraba reorganizar los estudios científicos. Para esto último, la Facultad se encontró con la falta de especialistas en la zona y se procuró buscar candidatos en Buenos Aires y La Plata, varios de los cuales rechazaron el contrato de trabajo ofrecido. En respuesta al pedido de esa institución, Gallardo envió un telegrama recomendando una señorita - a Petrocchi - que reunía las condiciones didácticas y la preparación requerida. Esto fue tratado en una reunión del cuerpo de académicos de la Facultad cordobesa, donde uno de ellos cuestionó si "no sería 
Ni solas ni resignadas

inconveniente el sexo para el desempeño de estas funciones". ${ }^{14}$ Después de un debate, se acordó que si la candidata reunía las condiciones didácticas y preparación requeridas, el "sexo" no podía ser un inconveniente para dirigir la cátedra. Sin embargo, esta entomóloga no fue contratada ni tampoco se consiguió un profesor de zoología hasta varios años después. En el campo de las ciencias naturales, como hemos señalado anteriormente, recién en la década de 1930 algunas mujeres comienzan a formar parte del profesorado universitario.

En el área de las ciencias geológicas, Edelmira Inés Mórtola (1894-1973) fue la primera egresada de ciencias naturales en presentar una tesis. En 1919 fue incorporada como ayudante en la Dirección de Minas, Geología e Hidrología del Ministerio de Agricultura. Entre 1907 y 1922 esta repartición estuvo bajo la dirección del ingeniero en minas, Enrique Hermitte (1871-1955), quien también enseñaba mineralogía, petrografía y geología en la Universidad de Buenos Aires, donde estudió Mórtola y se graduaron los primeros geólogos del país. Por intermedio de Hermitte se inició una estrecha relación entre ambos centros y gracias a lo cual los primeros alumnos de geología tuvieron oportunidad de especializarse y realizar sus primeras experiencias profesionales bajo la dirección de los investigadores alemanes que trabajaban en la Dirección. (Camacho, 1971, 2001, 2004) A partir de estos contactos, Mórtola realizó un estudio sobre rocas alcalinas básicas de Chubut recolectadas por el geólogo alemán Juan Keidel (1877-1954) en 1917, investigación que presentó como tesis doctoral en 1920. En esa Dirección trabajó hasta 1923 y al año siguiente ingresa como jefe de trabajos prácticos en la cátedra de Hermitte de la Facultad de Ciencias Exactas Físicas y Naturales de Buenos Aires, donde se ocupó de reorganizar las colecciones mineralógicas, petrográficas y paleontológicas de esta institución. Años más tarde se desempeñó como profesora adjunta de

${ }^{14}$ Las actas de esa reunión se publicaron en la sección oficial de la Revista de la Universidad Nacional de Córdoba, 1920, VII, n 8-10. 
mineralogía en la misma Facultad hasta 1948 y luego como profesora titular desde 1956 hasta su jubilación en 1960. Además, al igual que otras egresadas universitarias fue docente en el Liceo de Señoritas y el Instituto Nacional del Profesorado de Buenos Aires.

Como en el caso de esta geóloga, el ingreso a ciertas reparticiones estatales y otras instituciones científicas se realizaba a través de contactos personales y especialmente a través de los nexos establecidos entre profesores universitarios que dirigirían o trabajaban en algunos de esos centros. Entre ellos, se puede mencionar a Martín Doello Jurado, un egresado y profesor del Instituto Nacional del Profesorado Secundario, vinculado también como docente a la Facultad de Ciencias Exactas, Físicas y Naturales de Buenos Aires y al Museo Argentino de Ciencias Naturales de Buenos Aires, donde se ocupó de las colecciones de moluscos e invertebrados marinos y luego asumió como director entre 1924 y 1946. En esta institución, Doello Jurado comenzó a reclutar estudiantes, graduados y profesores en ciencias naturales para estudiar y catalogar determinadas colecciones, inicialmente las relacionadas con ciertos grupos de invertebrados marinos recolectados en sus expediciones. Así ingresa como ayudante, la uruguaya Deidamia Giambiagi (?-1947), luego de haberse doctorado en la Facultad de Ciencias Exactas, Físicas y Naturales de Buenos Aires en 1920 con una tesis sobre isópodos (un grupo de crustáceos) dirigida por Angel Gallardo. Paralelamente también ejerció la docencia secundaria y publicó trabajos científicos sobre foraminíferos fósiles, crustáceos y otros invertebrados actuales, actividad que continuó realizando después de casarse. Se destacó como especialista en isópodos terrestres y de los mares argentinos, estudiando y dando a conocer los ejemplares nuevos pertenecientes a las colecciones del Museo de Buenos Aires y las recolectadas en la Estación Marina de Quequén. Ganó el premio Strobel por su tesis y en 1935 el concurso de la cátedra de biología general y citología en la Universidad de Buenos Aires. 
Ni solas ni resignadas

En la misma época que ingresa Deidamia Giambiagi al Museo de Buenos Aires, Irene Bernasconi (1896-1989) se incorporó como "adscripta" honoraria. Nacida en La Plata, cursó sus estudios secundarios en el Liceo de Señoritas de la Capital Federal y en 1918 egresó del Instituto Nacional del Profesorado como Profesora de Ciencias Naturales. Mientras se desempeñó como docente en varios colegios secundarios, Bernasconi trabajó como encargada ad-honorem de las colecciones de equinodermos actuales y fósiles del Museo, las reunidas por Doello Jurado y otros naturalistas viajeros enviados por la institución, así como recolectadas por los buques de la Armada. Posteriormente, investigadores extranjeros $e$ instituciones de otros países le enviaron ejemplares para su determinación y estudio o la invitaron para catalogar colecciones. Además, completó sus monografías con ejemplares de la costa atlántica reunidos por ella en sus viajes privados, ya que en las primeras décadas del siglo XX las mujeres no participaron oficialmente en expediciones marítimas, las cuales se realizaron principalmente en buques de la Armada y compañías pesqueras. Las vinculaciones que mantuvo con la Marina y las comisiones de exploración marítima y antárticas, le permitieron participar con 72 años en la Campaña Antártica 1968/1969, junto con otras tres profesoras adscriptas al Museo de Buenos Aires, siendo las primeras mujeres argentinas en viajar al continente antártico. En esa época también llegó a ser investigadora del CONICET. Se destacó como especialista en equinodermos y otros organismos marinos, dando a conocer varias especies nuevas y publicando más de 70 trabajos sobre estos temas. Asimismo, mantuvo conexiones con diversos organismos nacionales $e$ internacionales de investigación marina, recibiendo varias distinciones como el Premio "Eduardo Holmberg" otorgado por la Academia de Ciencias Exactas, Físicas y Naturales de Buenos Aires en 1947 y la membresía del Instituto Rey Alberto de Bélgica, entre otros reconocimientos.

A través de estos itinerarios femeninos, que por cierto no fueron los únicos que se registran en el período estudiado, hemos 
intentado dar cuenta de las posibilidades laborales y las modalidades de ingreso a las instituciones científicas que se abrieron para las naturalistas a principios del siglo XX. La década de 1910, iniciada en el marco de los debates sobre la capacidad y "naturaleza" de la mujer y la realización de dos congresos femeninos en Buenos Aires, donde el Congreso Feminista Internacional reconoció que "ninguna condición psíquica ni social hacen ineptas a las mujeres para entregarse a las investigaciones científicas como lo demuestran los ejemplos cada vez más numerosos..."15, marca un punto de inflexión en el ingreso de las mujeres argentinas en la actividad científica-académica. En la siguiente década, las mujeres logran la igualdad de los derechos civiles y algunas llegan a ser profesoras universitarias. Mientras la década 1930 va a registrar el acceso de las egresadas en ciencias naturales al profesorado universitario, la búsqueda de una formación postdoctoral en el extranjero y la presencia de las mujeres se registra en todas las disciplinas y la mayoría de las instituciones de las ciencias naturales, destacándose como especialistas en determinados grupos de organismos y área de estudios.

\section{Consideraciones generales}

En este trabajo hemos procurado examinar el contexto y condiciones de posibilidad para la emergencia de la primera generación de mujeres "profesionales" en el campo de las ciencias naturales en la Argentina. En relación con ello, el acceso de las mujeres a los estudios superiores y una formación universitaria, constituye un punto importante para comprender la inserción de las mujeres en la vida científica y las "condiciones de partida" para su ingreso en la carrera profesional de la ciencia. El contacto personal con naturalistas de la época que se desempeñaban como

${ }^{15}$ Primer Congreso Femenino Internacional de La República Argentina. Historia, Actos y Trabajos. Buenos Aires, Imprenta Ceppi, 1911. 
Ni solas ni resignadas

profesores en la universidad y en otros ámbitos de formación de profesores en ciencias naturales, permitió que estos conocieran sus habilidades, capacidades, intereses y las reclutaran como ayudantes y colaboradoras en museos, laboratorios universitarios, institutos científicos y oficinas estatales de investigación aplicada. El acceso de las mujeres a la educación científica superior, permitió que algunas de ellas, sobre todo las que no provenían de familias tradicionales o de sectores científicos, pudieran ingresar en los circuitos de sociabilidad y las redes formales e informales de intercambio de información y materiales, así como acceder a publicar en las revistas científicas locales. Por otra parte, la existencia de becas y ayudantías abrió posibilidades y también ofreció una posición asalariada para aquellas alumnas - y a estudiantes varones - que no contaban con suficientes medios privados para emprender una carrera científica.

Remarquemos que esto no implicó que las mujeres gozaran de las mismas prerrogativas que sus colegas masculinos para desarrollar una carrera académica, y de hecho, en general tendieron a permanecer en los cargos más bajos de las jerarquías institucionales o vinculados a actividades administrativas como secretarias y auxiliares. Asimismo, no faltaron ocasiones en las que de forma explicita o implícita fueran excluidas de ciertos cargos o actividades, especialmente aquellas vinculadas con el trabajo de campo que para algunos no parecía compatibles con la "condición femenina". Como han analizado historiadoras y sociólogas en otros contextos, varias de las primeras naturalistas argentinas se encontraron inicialmente relegadas a tareas específicas dentro de la actividad de cada disciplina, tareas auxiliares fundamentales pero con menor reconocimiento en la producción de conocimientos, tales como computar datos, clasificar y catalogar especimenes, preparar ejemplares o confeccionar los gráficos que acompañan las publicaciones científicas. Los puestos rentados para cumplir con estas tareas a las que accedieron las mujeres hablan de una mayor burocratización de las prácticas científicas y de cómo estereotipos sobre "la naturaleza femenina" y cierta 
división sexual de tareas operaron como mecanismos no explícitos en la asignación de labores. Al mismo tiempo, esta primera generación de naturalistas también se topó en el desarrollo de sus carreras con un "techo de cristal" en las jerarquías académicas e institucionales, donde inicialmente permanecieron en los estratos inferiores a pesar de que algunas podían presentar las mismas credenciales y habilidades que sus colegas masculinos que ascendieron a posiciones más elevadas.

En algunos trabajos se ha señalado que otro tipo de exclusión de las mujeres en el ámbito científico se dió en los circuitos formales $e$ informales de intercambio de información y materiales, lo que implicaba un menor acceso al capital social y a redes de relaciones y contactos. Sin embargo, la lectura de los trabajos científicos publicados por las primeras naturalistas muestra que tuvieron acceso a laboratorios, colecciones de estudio y bibliografía especializada, ya sea por sus relaciones familiares, sus estudios universitarios o su colaboración en instituciones científicas. Las trayectorias de estas mujeres en los inicios del siglo $\mathrm{XX}$ muestra que lejos de estar solas y resignadas, recibieron el apoyo de profesores y figuras importantes del campo disciplinar, $y$ al mismo tiempo, utilizaron las estrategias, conexiones y circuitos disponibles para acceder a una formación universitaria y a la adscripción institucional necesaria para disponer de material para investigar y publicar trabajos científicos, por los que varias recibieron premios y reconocimientos de sus colegas masculinos.

\section{Referencias bibliograficas}

BARRANCOS, Dora. Inclusión/Exclusión. Historia con mujeres. Buenos Aires, Fondo de Cultura Económica, 2002.

- Itinerarios científicos femeninos a principios del siglo XX: solas, pero no resignadas. En: MONTSERRAT, Marcelo. (comp.) La ciencia en la Argentina entre siglos. Buenos Aires, Manatial, 2000, pp.127-144. 
Ni solas ni resignadas

BIANCHI, Susana. ¿Historia de mujeres o mujeres en la historia? In: AAVV. Feminismo, Ciencia, Cultura y Sociedad. Buenos Aires, Humanitas, 1992, pp.17-33.

CAMACHO, H. La primera generación de Geólogos Argentinos. El Carnotaurus, vol. 51, Buenos Aires, Museo Argentino de Ciencias Naturales Bernardino Rivadavia, 2004, pp.2-3. Versión on line: http://www.macn.secyt.gov.ar/boletin/jun04.pdf

. Las ciencias geológicas en la Argentina, hasta 1939. Saber y Tiempo 12, 2001, pp.177-220.

Las ciencias naturales en la Universidad de Buenos Aires. Estudio histórico, Buenos Aires, Eudeba, 1971.

CANDIOTI, Marcial R. Bibliografía doctoral de la Universidad de Buenos Aires y catálogo cronológico de las Tesis en su primer centenario 1821-1920. Revista de la Universidad de Buenos Aires, XLIV-XLV, $\mathrm{n}^{\circ}$ 146, Buenos Aires, Universidad de Buenos Aires, 1920, pp.7051116.

CASTELlANOS, Zulma A. María Isabel Hylton Scott de Birabén 18891990. Neotrópica 36 (95), La Plata, 1990, pp.3-4.

DE SANTIS, Luis. Entomología. Evolución de las Ciencias en la Republica Argentina 1923-1972, tomo XII. Buenos Aires, Sociedad Científica Argentina, 1992.

DEAUTIER, Enrique A. Dra. Juana M. Petrocchi. Physis VII, 27, Buenos Aires, Sociedad Argentina de Ciencias Naturales, 1925, pp.417-20. Juana Miguela Petrocchi. Reseña Biográfica. Revista de la Sociedad Entomológica Argentina (III), 1930-31, pp.271-274.

FACULTAD DE AGRONOMÍA, UNLP. Nómina de titulados y egresados desde la fundación de la Facultad en 1883 hasta el 31 de diciembre de 1925. La Plata, 1926.

GARCíA, Susana Valeria. El Museo de La Plata y la divulgación científica en el marco de la extensión universitaria (1906-1930). Tesis doctoral. Facultad de Ciencias Naturales y Museo, Universidad Nacional de La Plata, 2004a.

Miguel Fernández y el proyecto científico-educativo del Laboratorio de Zoología del Museo de La Plata. Saber y Tiempo 17, Buenos Aires, 2004b, pp.99-126. 
La promoción de la actividad científica en los inicios de la Universidad Nacional de La Plata (1906-1918). Pensamiento Universitario (XI), Buenos Aires, 2004c, pp.43-47.

GRACIANO, Osvaldo. Universidad y Economía Agroexportadora: el perfil profesional de los ingenieros agrónomos 1910-1930. In: GIRBALBLANCHA, Noemí. (comp.) Agro, Universidad y enseñanza. Dos momentos de la Argentina rural (1910-1955). La Plata, Editorial de la UNLP, 1998, pp.13-72.

HICKEN, Cristobal. Evolución de las ciencias en la República Argentina. VII Estudios Botánicos. Buenos Aires, Sociedad Científica Argentina, 1923.

KOHN LONCARICA, Alfredo y Sánchez, Norma Isabel. La mujer en la medicina argentina: las médicas de la primera década del siglo XX. Saber y Tiempo (2), Buenos Aires, Centro de Estudios de Historia de la Ciencia José Babini, 1996, pp.113-138.

y Sánchez, Norma Isabel. La mujer en la medicina argentina: las médicas de la segunda década del siglo XX. Saber y Tiempo (9), Buenos Aires, Centro de Estudios de Historia de la Ciencia José Babini, 2000, pp.89-107.

. Cecilia Grierson. Vida y obra de la primera médica argentina. Buenos Aires, Stlcograf, 1976.

LAFONE QUEVEDO, Samuel. Memoria del director del Instituto del Museo correspondiente al año 1917. Boletín de la Universidad Nacional de La Plata (I, 3), La Plata, 1918, pp.106-122.

Memoria presentada al Congreso Nacional por el Ministro de Justicia e Instrucción Pública. Buenos Aires, Talleres de la Penitenciaria Nacional, 1926.

PALERMO, Alicia Italí. Mujeres profesionales que ejercieron en la Argentina en el siglo XIX, Convergencia (XII 38), México, Universidad Autónoma del Estado de México, 2005, pp.59-79.

PARODI, Lorenzo. Ciento Cincuenta Años de Botánica en la República Argentina. Boletín de la Sociedad Argentina de Botánica (IX), Buenos Aires, 1961.

PINKASZ, Daniel. Orígenes del profesorado secundario en la Argentina. Tensiones y conflictos. In: BRASLAVSKY, Cecilia y BIRGIN, Alejandra. 
Ni solas ni resignadas

Formación del Profesorado. Impacto, pasado y presente. Buenos Aires, Miño Dávila, 1992, pp.59-82.

PODGORNY, Irina. Egresados del país: es necesario reaccionar! Ciencia Hoy (6), Buenos Aires, 1996, pp.60-64.

Primer Congreso Femenino Internacional de La República Argentina. Historia, Actos y Trabajos. Buenos Aires, Imprenta Ceppi, 1911.

PYENSON, Lewis. Cultural Imperialism and Exact Sciences: German Expansion Overseas. New York, Peter Lang, 1985.

Revista de la Universidad Nacional de Córdoba, VII, n’s 8-10, 1920.

RICHMOND, Marsha L. "A Lab of One's Own". The Balfour Biological Laboratory for Women at Cambridge University, 1884-1914. Isis (88), 1997, pp.422-455.

ROSSITER, Margaret W. "Women's Work" in Science, 1880-1910. Isis (70), 1980, pp.381-398.

. Women Scientists in America: Struggles and Strategies to 1940. Baltimore, The Johns Hopkins Press, 1982.

TOFFOLI DE MATEOS, Myrta y SPEGAZZINI, Etile. Carolina Etile Spegazzini: Farmacéutica Naturalista y Química. Acta Farmacéutica Bonaerense (6, 2). 1987, pp.128-135. (versión on-line: www.biol. unlp.edu.ar/historiafarmacia)

VON REICHENBACH, Cecilia. Margrete Heiberg-Bose: a Danish Chemist Pioneer of Physics in Argentina. In: RODRÍGUEZ-SALA, María Luisa y ZUBIETA GARCÍA, Judith. (comp.) Mujeres en la ciencia y la tecnología: Hispanoamérica y Europa. México, 2005. 